\title{
Technical note: Evaluation of procedures for analyzing ration sorting and rumen digesta particle size in dairy cows ${ }^{1}$
}

\author{
D. D. Maulfair and A. J. Heinrichs ${ }^{2}$ \\ Department of Dairy and Animal Science, The Pennsylvania State University, University Park 16802
}

\begin{abstract}
Collecting total mixed ration (TMR) samples throughout the day to measure sorting activity of dairy cows may cause changes to the sorting behavior of cows or may make it more difficult to elucidate effects of sorting on TMR particle size distributions. Also, forage particle size research commonly includes analysis of the solid portion of rumen digesta for particle size distribution after digesta has been squeezed through several layers of cheesecloth. Therefore, the first objective of this experiment was to determine if collecting TMR samples throughout the day affected sorting behavior of cows and resulted in a different particle size distribution than when TMR was not artificially altered during the day. The second objective of this experiment was to determine if squeezing rumen digesta samples through cheesecloth changed particle size distribution when analyzed by a wet sieving technique. It was determined that small, significant differences existed in particle size distribution between the 2 sampling methods of TMR for sorting behavior. These differences were more likely to occur at time points later in the day. This resulted in small changes in sorting indices calculated from these data; sampling and mixing TMR throughout the day reduced the degree of sorting. Squeezing rumen digesta through 4 layers of cheesecloth had no effect on particle size distribution of particles $>0.15 \mathrm{~mm}$ but reduced the amount of rumen fluid-associated dry matter contained in the sample.
\end{abstract}

Key words: dairy cow, feeding behavior, particle size, sorting

When collecting TMR samples to analyze ration sorting it is necessary to thoroughly mix the remaining TMR to collect representative samples. Several studies (Kononoff et al., 2003; Leonardi and Armentano, 2003; Hosseinkhani et al., 2008) have sampled the remaining

\footnotetext{
Received January 19, 2010.

Accepted April 3, 2010.

${ }^{1}$ This research was a component of NC-1042; Management Systems to Improve the Economic and Environmental Sustainability of Dairy Enterprises.

${ }^{2}$ Corresponding author: ajh@psu.edu
}

TMR several times throughout the day. This method may lead to incorrect conclusions about sorting because any sorting that had occurred up to sampling time would be nullified during sample collection. It has not been determined if mixing TMR to take samples affects further ration sorting behavior of dairy cows. Therefore, the first objective of this experiment was to determine if collecting TMR samples throughout the day affected sorting behavior of cows and resulted in a different particle size distribution than when TMR was not artificially altered during the day. When taking rumen samples from fistulated dairy cows during feeding studies, it is common procedure to squeeze rumen digesta through several layers of cheesecloth to obtain the fluid fraction for analysis (Kononoff et al., 2003). The solid fraction retained on the cheesecloth sometimes is used for particle size distribution analysis via wet sieving. However, it is not known if squeezing through cheesecloth affects particle size distribution of the solid fraction. Therefore, the second objective of this experiment was to determine if squeezing rumen digesta samples through cheesecloth changed the particle size distribution when analyzed by a wet sieving technique.

Data for this paper were collected during the final period of a feeding trial designed to study the effects of varying forage particle size on ration sorting in lactating dairy cows (Maulfair et al., 2010). Cows were cared for and maintained according to a procedure approved by The Pennsylvania State University Institutional Animal Care and Use Committee. Eight lactating, multiparous, Holstein cows averaging $90 \pm 32$ DIM, weighing $642 \pm 82 \mathrm{~kg}$, and with parity of $2.25 \pm 0.46$ (mean \pm SD) were randomly assigned to replicated $4 \times$ 4 Latin squares; 1 square of cows was rumen fistulated. The periods were $21 \mathrm{~d}$ in length, with a 13-d adaptation period followed by an 8-d collection period. During each of the 4 periods, cows were fed 1 of 4 rations that contained identical feed ingredients and proportions. Ration ingredients and their percentage of ration DM were corn silage (29.4), alfalfa haylage (17.6), grass hay (11.8), ground corn (22.9), roasted soybeans (6.7), canola meal (5.7), heat-treated soybean meal (3.2), mineral/vitamin mix (2.4), and salt (0.3). Rations con- 
tained $15.9 \% \mathrm{CP}, 34.0 \% \mathrm{NDF}$, and $1.65 \mathrm{Mcal} / \mathrm{kg} \mathrm{NE}$ and varied only in chop length of the dry grass hay included in the ration. Particle sizes (geometric mean $\pm \mathrm{SD}, \mathrm{mm})$ of the rations were as follows: short (4.46 $\pm 3.02)$, medium $(5.10 \pm 3.56)$, long $(5.32 \pm 3.92)$, and extra long $(5.84 \pm 4.39)$ as determined by ASABE (2007). All diets were mixed separately using an I. H. Rissler model 1050 TMR mixer (E. Rissler Mfg. LLC, New Enterprise, PA). Animals were housed in individual stalls in a mechanically ventilated barn, milked twice a day at 0700 and $1900 \mathrm{~h}$, and fed once a day at approximately $0730 \mathrm{~h}$ for ad libitum consumption and a $10 \%$ refusal rate to allow for maximum opportunity to sort the ration. Feed was pushed up but not mixed at 1230,1730 , and $2400 \mathrm{~h}$. All rations were balanced to meet or exceed NRC (2001) requirements, and water was available ad libitum.

To examine whether mixing and sampling remaining feed affected sorting behavior of dairy cows, TMR samples were taken on d 20 and 21 at $0,2,4,8,12$, 16 , and $24 \mathrm{~h}$ after feeding (hereafter, the mixed sampling scheme). In addition, samples were taken at 0 and $8 \mathrm{~h}$ (d 19 and 22); 0 and $16 \mathrm{~h}$ (d 23 and 24); and 0 and $24 \mathrm{~h} \mathrm{(d} 25$ and 26) after feeding (hereafter, the unmixed sampling scheme). During the study, average DMI was $25.89 \pm 0.88 \mathrm{~kg} / \mathrm{d}$ and refusals averaged 12.21 $\pm 0.70 \%$ of DMI; DMI and refusals were not different between treatments $(P>0.24$ and 0.22 , respectively). At each sampling point TMR was removed from the feed bunk, weighed, thoroughly mixed, sampled, and then returned to the cow, which is standard procedure in feeding studies. All samples were sieved in the American Society of Agriculture Engineers forage particle separator, which can determine 6 particle fractions $(>26.9,>18.0,>8.98,>5.61,>1.65$, and $<1.65$ mm; screen diagonal; (ASABE., 2007). Geometric mean particle length $\left(\mathbf{X}_{\mathrm{gm}}\right)$ and standard deviation of particle length $\left(\mathbf{S}_{\mathrm{gm}}\right)$ were calculated according to the ASABE (2007) procedure. Because $>1 \%$ of material was retained on the top screen, 3 samples of each diet were randomly selected, and all particles retained on the top screen were measured manually (with a ruler) before drying. Measured mean particle sizes for the top screen were $118.8 \pm 3.6,105.7 \pm 9.1,84.5 \pm 2.6$, and $74.8 \pm 6.6$ (mean $\pm \mathrm{SD}, \mathrm{mm}$ ) for the extra long, long, medium, and short diets, respectively. Whole samples were then placed in a forced air oven at $55^{\circ} \mathrm{C}$ for $48 \mathrm{~h}$ to determine DM content. Sorting indices based on refus-

Table 1. Percentage of uneaten TMR particles (DM basis) retained on sieves at 8 -h intervals after feeding when sampled by 2 different procedures (mixed and unmixed)

\begin{tabular}{|c|c|c|c|c|}
\hline Item $^{1}$ & Mixed $^{2}$ & Unmixed $^{3}$ & SE & $P$-value \\
\hline \multicolumn{5}{|l|}{ Hour 0} \\
\hline $26.9 \mathrm{~mm}$ & 6.72 & 5.35 & 1.29 & 0.42 \\
\hline $18.0 \mathrm{~mm}$ & 4.17 & 3.80 & 0.26 & 0.17 \\
\hline $8.98 \mathrm{~mm}$ & 20.3 & 20.4 & 0.43 & 0.89 \\
\hline $5.61 \mathrm{~mm}$ & 21.2 & 20.6 & 0.33 & 0.27 \\
\hline $1.65 \mathrm{~mm}$ & 23.7 & 24.9 & 0.65 & 0.10 \\
\hline Pan & 23.9 & 24.9 & 0.76 & 0.24 \\
\hline \multicolumn{5}{|l|}{ Hour 8} \\
\hline $26.9 \mathrm{~mm}$ & 8.15 & 5.95 & 1.54 & 0.28 \\
\hline $18.0 \mathrm{~mm}$ & 4.71 & 4.17 & 0.29 & 0.10 \\
\hline $8.98 \mathrm{~mm}$ & 21.9 & 21.3 & 0.53 & 0.44 \\
\hline $5.61 \mathrm{~mm}$ & 20.9 & 21.2 & 0.41 & 0.58 \\
\hline $1.65 \mathrm{~mm}$ & 22.7 & 23.3 & 0.74 & 0.46 \\
\hline Pan & 21.7 & 24.0 & 0.87 & 0.03 \\
\hline \multicolumn{5}{|l|}{ Hour 16} \\
\hline $26.9 \mathrm{~mm}$ & 10.9 & 11.6 & 1.54 & 0.72 \\
\hline $18.0 \mathrm{~mm}$ & 4.65 & 6.38 & 0.29 & $<0.01$ \\
\hline $8.98 \mathrm{~mm}$ & 22.1 & 26.6 & 0.53 & $<0.01$ \\
\hline $5.61 \mathrm{~mm}$ & 21.4 & 20.6 & 0.41 & 0.17 \\
\hline $1.65 \mathrm{~mm}$ & 21.1 & 21.1 & 0.74 & 0.99 \\
\hline Pan & 19.9 & 13.7 & 0.87 & $<0.01$ \\
\hline \multicolumn{5}{|l|}{ Hour 24} \\
\hline $26.9 \mathrm{~mm}$ & 12.0 & 17.1 & 1.54 & 0.01 \\
\hline $18.0 \mathrm{~mm}$ & 5.70 & 5.19 & 0.29 & 0.12 \\
\hline $8.98 \mathrm{~mm}$ & 24.2 & 22.0 & 0.53 & $<0.01$ \\
\hline $5.61 \mathrm{~mm}$ & 21.3 & 19.8 & 0.41 & $<0.01$ \\
\hline $1.65 \mathrm{~mm}$ & 19.6 & 19.0 & 0.74 & 0.55 \\
\hline Pan & 17.2 & 16.9 & 0.87 & 0.72 \\
\hline
\end{tabular}

${ }^{1}$ Pore size of screens.

${ }^{2} \mathrm{TMR}$ was mixed, sampled, and returned to cow at $2,4,8,12,16$, and $24 \mathrm{~h}$ after feeding.

${ }^{3} \mathrm{TMR}$ was not mixed until sample collections at the respective time point. 
Table 2. Geometric mean particle length of uneaten TMR and sorting index of the consumed $\operatorname{diet}^{1}$ obtained with 2 different sampling procedures (mixed and unmixed)

\begin{tabular}{|c|c|c|c|c|}
\hline Item $^{2}$ & Mixed $^{3}$ & Unmixed $^{4}$ & $\mathrm{SE}$ & $P$-value \\
\hline \multicolumn{5}{|l|}{ Hour 0} \\
\hline $\mathrm{X}_{\mathrm{gm}}, \mathrm{mm}$ & 4.87 & 4.58 & 0.57 & 0.71 \\
\hline $\mathrm{S}_{\mathrm{gm}}, \mathrm{mm}$ & 3.72 & 3.59 & 0.07 & 0.13 \\
\hline \multicolumn{5}{|l|}{ Hour 8} \\
\hline $\mathrm{X}_{\mathrm{gm}}, \mathrm{mm}$ & 5.63 & 4.90 & 0.70 & 0.45 \\
\hline $\mathrm{S}_{\mathrm{gm}}, \mathrm{mm}$ & 3.82 & 3.65 & 0.08 & 0.12 \\
\hline Index $^{6}$ & 0.89 & 0.95 & 0.03 & 0.17 \\
\hline \multicolumn{5}{|l|}{ Hour 16} \\
\hline $\mathrm{X}_{\mathrm{gm}}, \mathrm{mm}$ & 6.72 & 8.40 & 0.70 & 0.08 \\
\hline $\mathrm{S}_{\mathrm{gm}}, \mathrm{mm}$ & 3.95 & 3.60 & 0.08 & $<0.01$ \\
\hline Index & 0.92 & 0.95 & 0.02 & 0.17 \\
\hline \multicolumn{5}{|l|}{ Hour 24} \\
\hline $\mathrm{X}_{\mathrm{gm}}, \mathrm{mm}$ & 7.84 & 10.1 & 0.70 & 0.02 \\
\hline $\mathrm{S}_{\mathrm{gm}}^{\circ}, \mathrm{mm}$ & 3.85 & 4.04 & 0.08 & 0.07 \\
\hline Index & 0.93 & 0.89 & 0.01 & $<0.01$ \\
\hline
\end{tabular}

als were calculated for particle size fractions at 8,16 , and $24 \mathrm{~h}$ after feeding. Actual intake of each particle fraction was divided by expected intake of each particle fraction (Leonardi and Armentano, 2003). Values >1 indicate selective consumption and values $<1$ indicate selective refusal of the DM retained on an individual sieve. Additionally, $\mathrm{X}_{\mathrm{gm}}$ sorting indices were calculated for the same time points by dividing the $\mathrm{X}_{\mathrm{gm}}$ of TMR consumed up to each time point by $\mathrm{X}_{\mathrm{gm}}$ at time 0 . Values $>1$ indicate that cows were consuming rations with longer particle size and values $<1$ indicate that cows were consuming rations with shorter particle size than the diets fed. Statistical analysis was conducted using the MIXED procedure of SAS (SAS Institute, 2006). The model included sampling method, time, and diet as fixed effects, cow as a random effect, and the interaction of sampling method and time. All denominator degrees of freedom for $F$-tests were calculated according to Kenward and Roger (1997). All data are presented as least squares means, and sampling method effects are considered significant when $P<0.05$ and a trend when $P<0.10$.

It was found that TMR did differ slightly when particle distribution was expressed by individual screens (Table 1). There were some small, significant differences in several of the particle fractions, and sampling method generated larger, more significant differences as the time after feeding increased. In general, where there were significant differences, the unmixed sampling protocol had a longer particle size than did the mixed protocol. When particle distribution of the unconsumed
TMR was expressed as geometric mean particle length (Table 2) there were no differences for 0 and $8 \mathrm{~h}$. However, there was a trend at $16 \mathrm{~h}$ for unmixed to be longer than mixed, and at $24 \mathrm{~h}$ samples of remaining TMR collected with the unmixed protocol had significantly longer particles than those collected under the mixed sampling scheme. In Table 2 the $\mathrm{X}_{\mathrm{gm}}$ and $\mathrm{S}_{\mathrm{gm}}$ values are from the unconsumed TMR or what was left in the feed bunk at that time point. They are the same data as are found in Table 1 but converted from individual screens to an average particle size. There was a significant linear contrast for the sampling procedure by time interaction, indicating that the $\mathrm{X}_{\mathrm{gm}}$ of the uneaten diet increased to a greater extent for unmixed than for mixed with increasing time. When sorting index was calculated using all 6 particle fractions, virtually no significant differences in sorting were found between sampling methods at any of the time points (data not shown). Using the sorting index calculated with $\mathrm{X}_{\mathrm{gm}}$ (Table 2), cows were eating shorter rations than they were fed when analyzed using both sampling procedures at all time points. There were no differences at 8 and 16 $\mathrm{h}$, but at $24 \mathrm{~h}$, the unmixed sampling procedure resulted in a lower selection index than the mixed sampling procedure. Again, the linear contrast for the sampling procedure by time interaction was significant because sorting index increased for the mixed and decreased for the unmixed sampling method over time. Diet effects on sorting behavior were found to be significant and are discussed in Maulfair et al. (2010). Based on these results, mixing the TMR several times throughout the 
Table 3. Percentage of rumen digesta particles (DM basis) retained on sieves after wet sieving when digesta samples were prepared with or without being squeezed through cheesecloth

\begin{tabular}{lcccc}
\hline Item $^{1}$ & Squeezed & Nonsqueezed & SE & $P$-value \\
\hline $9.5 \mathrm{~mm}$ & 17.3 & 16.4 & 0.96 & 0.58 \\
$6.5 \mathrm{~mm}$ & 6.56 & 6.63 & 0.24 & 0.84 \\
$3.35 \mathrm{~mm}$ & 18.4 & 18.7 & 0.62 & 0.76 \\
$1.18 \mathrm{~mm}$ & 20.4 & 20.9 & 0.27 & 0.21 \\
$0.6 \mathrm{~mm}$ & 12.9 & 12.8 & 0.18 & 0.63 \\
$0.15 \mathrm{~mm}$ & 24.5 & 24.6 & 0.38 & 0.90 \\
Fluid-assoc/solid-assoc $^{2}$ & 34.9 & 46.2 & 1.04 & $<0.01$ \\
\hline
\end{tabular}

${ }^{1}$ Pore size of screens.

${ }^{2}$ Rumen fluid-associated DM per unit of solid-associated DM.

day to obtain a representative sample caused the particle size of the unconsumed TMR to be smaller and biased conclusions about sorting behavior toward less sorting than what actually occurred.

To determine if squeezing rumen digesta through cheesecloth affected particle size distribution obtained via wet sieving, rumen samples were taken on d 15 at $0.0,1.5,3.5,5.5,8.5,11.5,14.5,18.0,21.5$, and 24.5 $\mathrm{h}$ after feeding. Samples were taken from 5 rumen locations (dorsal, ventral, cranial, caudal, and medial areas), mixed thoroughly, and then separated into 2 equal parts. One part was squeezed though 4 layers of cheesecloth, and the solid fraction retained on the cheesecloth was stored in a $-20^{\circ} \mathrm{C}$ freezer. The second part was stored the same way, but without the initial squeezing. To determine particle size distribution, the 2 samples were then wet sieved using a procedure modified from Beauchemin et al. (1997). Subsamples (approximately $30 \mathrm{~g}$ ) were placed on a series of stacked sieves (sizes 0.15, 0.6, 1.18, 3.35, 6.7, $9.5 \mathrm{~mm}$; VWR, Arlington Heights, IL) contained in a Retsch AS 200 Control sieve shaker (Retsch, Haan, Germany) and sieved in duplicate. The samples were sieved for $10 \mathrm{~min}$ at $2.5 \mathrm{~mm}$ amplitude with the cold water flow rate at approximately 1.5 to $2.0 \mathrm{~L} / \mathrm{min}$ to ensure particles were separated thoroughly. Contents retained on the sieves were rinsed with cold water into a funnel with rumen in situ bags $(5 \times 10 \mathrm{~cm}, 53-\mu \mathrm{m}$ pore size; Ankom, Macedon, NY) attached to the stem to collect the sample. Bags were then dried in a forced air oven at $55^{\circ} \mathrm{C}$ for 24 $\mathrm{h}$ and weighed to determine DM retained on each sieve. A portion of each sample was also dried at $55^{\circ} \mathrm{C}$ for 24 $\mathrm{h}$ in a forced air oven without sieving to determine the DM content of the original sample. The rumen fluidassociated fraction of the sample was calculated as the DM lost during the sieving and drying process. Statistical analysis was conducted using the MIXED procedure (SAS Institute, 2006). The model included sampling method, time, and diet as fixed effects and cow as a random effect. All denominator degrees of freedom for $F$-tests were calculated according to Kenward and
Roger (1997). All data are presented as least squares means, and treatment effects are considered significant when $P<0.05$ and a trend when $P<0.10$.

No significant differences were found between the 2 sampling techniques for any of the fractions retained on screens (Table 3). There was significantly more $(46.22$ vs. $34.87 \%$ ) rumen fluid-associated DM per unit of solid-associated DM for samples that were not squeezed through cheesecloth. If the proportion of rumen fluidassociated DM is important to the objective of the experiment, then rumen digesta should not be squeezed before wet sieving. However, if only particles retained on screens are of importance, using rumen digesta after squeezing will have no effect on results.

In conclusion, mixing TMR to take representative samples several times throughout the day had a small effect of decreasing the particle size of uneaten feed, which may lead to the conclusion that cows are sorting their ration to a lesser extent (when cows are sorting against longer particles and for shorter particles) than if sorting samples were taken only at the end of the sampling interval. In addition, squeezing rumen digesta through 4 layers of cheesecloth had no effect on particle fractions $>0.15 \mathrm{~mm}$, but it reduced the proportion of rumen fluid-associated DM per unit of solid-associated DM.

\section{ACKNOWLEDGMENTS}

Sincere appreciation is extended to Geoff Zanton (Penn State, University Park, PA) for statistical advice and support. This research was supported in part by agricultural research funds administered by The Pennsylvania Department of Agriculture.

\section{REFERENCES}

ASABE. 2007. Method of determining and expressing particle size of chopped forage materials by screening. ANSI/ASAE S242.1:663665 .

Beauchemin, K. A., L. M. Rode, and M. V. Eliason. 1997. Chewing activities and milk production of dairy cows fed alfalfa as hay, silage, or dried cubes of hay or silage. J. Dairy Sci. 80:324-333. 
Hosseinkhani, A., T. J. DeVries, K. L. Proudfoot, R. Valizadeh, D. M. Veira, and M. A. G. von Keyserlingk. 2008. The effects of feed bunk competition on the feed sorting behavior of close-up dry cows. J. Dairy Sci. 91:1115-1121.

Kenward, M. G., and J. H. Roger. 1997. Small sample inference for fixed effects from restricted maximum likelihood. Biometrics 53:983-997.

Kononoff, P. J., A. J. Heinrichs, and H. A. Lehman. 2003. The effect of corn silage particle size on eating behavior, chewing activities, and rumen fermentation in lactating dairy cows. J. Dairy Sci. $86: 3343-3353$.
Leonardi, C., and L. E. Armentano. 2003. Effect of quantity, quality, and length of alfalfa hay on selective consumption by dairy cows. J. Dairy Sci. 86:557-564.

Maulfair, D. D., G. I. Zanton, M. Fustini, and A. J. Heinrichs. 2010 Effect of feed sorting on chewing behavior, production, and rumen fermentation in lactating dairy cows. J. Dairy Sci. doi:10.3168/ jds.2010-3278

NRC. 2001. Nutrient Requirements of Dairy Cattle. 7th rev. ed. Natl. Acad. Sci., Washington, DC.

SAS Institute. 2006. SAS User's Guide: Statistics. Version 9.1.3. SAS Inst. Inc., Cary, NC. 